\title{
Peripheral Neural Mechanisms Determining the Orientation of Cylinders Grasped by the Digits
}

\author{
M. J. Dodson, ${ }^{1}$ A. W. Goodwin, ${ }^{1}$ A. S. Browning, ${ }^{2}$ and H. M. Gehring ${ }^{1}$ \\ ${ }^{1}$ Department of Anatomy and Cell Biology, University of Melbourne, Parkville, Victoria 3052, Australia, and 2Department of \\ Experimental Psychology, Oxford University, Oxford OX1 3UD, England
}

\begin{abstract}
When a human grasps a cylindrical object, feedback on the orientation of the cylinder with respect to the axes of the digits is crucial for successful manipulation of the object. We measured the ability of humans to discriminate the orientations of cylinders passively contacting the fingerpad. For a cylinder of curvature of $521 \mathrm{~m}^{-1}$ (radius, $1.92 \mathrm{~mm}$ ) subjects were able to discriminate, at the $75 \%$ level, orientation differences of $5.4^{\circ}$; for a less curved cylinder (curvature, $172 \mathrm{~m}^{-1}$; radius, $5.81 \mathrm{~mm}$ ) the difference limen decreased to $4.2^{\circ}$. The neural mechanisms underlying the determination of tactile orientation were investigated by recording the responses of single slowly adapting type I afferents (SAls) innervating the fingerpads of anesthetized monkeys. When cylinders were stepped across the receptive field of an SAI, the resulting response profiles were Gaussian in shape; the shape corresponded to the shape of the cylinder, increasing in height and decreasing in width for more curved cylinders. All SAls had the same underlying profile shape except for a multiplicative constant determined by the sensitivity
\end{abstract}

of the individual afferent. Thus it was possible to reconstruct the response of the population of active SAls in the fingerpad. Changing the orientation of the cylinder resulted in a rotation of the population response, but the change in angle of the population response was greater than the change in orientation of the cylinder. This discrepancy increased as the orientation of the cylinder moved closer to the orientation of the axis of the finger and was more pronounced for the less curved cylinder. Measured contact areas between the cylinders and the skin were elliptical, with orientations exceeding those of the cylinder; again the differences were greater for the less curved cylinder and for orientations closer to that of the finger axis. The human discrimination performance could be explained in terms of the SAI population responses.

Key words: tactile orientation; mechanoreceptor; cutaneous afferent; finger; grasp; shape; curvature; cylinder; skin mechanics
The evolution of the hand with its opposable thumb, independent finger movements (Tubiana, 1981), and high density of cutaneous innervation (Johansson and Vallbo, 1979) has led to dexterous manipulations that are of great importance to human existence. For appropriate motor control of the hand, the brain must have information about a number of parameters of the object being manipulated. Cylinders characterize a class of objects that we handle regularly, e.g., when writing with a pen or eating with chopsticks. A cylinder is defined completely by two parameters, its orientation and its curvature. At any one instant of time, the only source of information about the curvature of the cylinder (local shape), its position on the digits, and its orientation with respect to the digits is the population of mechanoreceptors in the skin contacting the object (Goodwin, 1997).

A number of studies have elucidated how the local shape of contacted objects may be relayed to the brain by the cutaneous mechanoreceptive afferents. LaMotte and Srinivasan used sinusoidally shaped steps, indenting them into and scanning them across monkeys' fingerpads (LaMotte and Srinivasan, 1987a,b; Srinivasan and LaMotte, 1987). They demonstrated that the shapes of the steps were represented in the responses of the cutaneous afferents and showed that these responses could ex-

Received Aug. 1, 1997; revised Oct. 16, 1997; accepted Oct. 20, 1997.

This work was supported by a grant from the National Health and Medical Research Council of Australia.

Correspondence should be addressed to A. W. Goodwin, Department of Anatomy and Cell Biology, University of Melbourne, Parkville, Victoria 3052, Australia Copyright (C) 1997 Society for Neuroscience $\quad 0270-6474 / 97 / 180521-\bullet \$ 05.00 / 0$ plain the human ability to discriminate such shaped steps. There have also been studies of the responses of cutaneous afferents to ellipsoids scanned over the fingerpad (LaMotte et al., 1994) and to cylindrical wavy surfaces scanned across the fingerpad (LaMotte and Srinivasan, 1996). Using spherical stimuli, Goodwin et al. (1991, 1995) and Wheat et al. (1995) showed that the reconstructed responses of the population of active slowly adapting type I afferents (SAIs), recorded in monkeys, accounted for their psychophysical measurements of the human capacity to discriminate the curvatures of the spheres and also their positions on the fingerpad. Determination of local shape, mainly in the context of robotics has also been the subject of a number of theoretical studies (Allen and Michelman, 1990; Berkemeier and Fearing, 1993; Reynaerts and van Brussel, 1993; Hahn, 1994).

Few studies have been related directly to the orientation of grasped objects. A number of investigators, measuring either human psychophysical performance or neural responses in monkeys, have explored the orientation of patterns of vibrating dots (Schneider et al., 1986; Kops and Gardner, 1996) or the orientation of stimuli scanned across the skin (Costanzo and Gardner, 1980; Warren et al., 1986; Essick and Edin, 1995; Ruiz et al., 1995). In addition, tactile resolution is often measured by requiring the subject to distinguish between two possible orthogonal orientations of edges or gratings (Essock et al., 1992; Van Boven and Johnson, 1994; Stevens and Patterson, 1995). Lechelt (1992) investigated cylinder orientation directly and, using a bimanual comparison task, showed that humans could discriminate orientation differences of $10^{\circ}$. LaMotte and Srinivasan (1993) have 

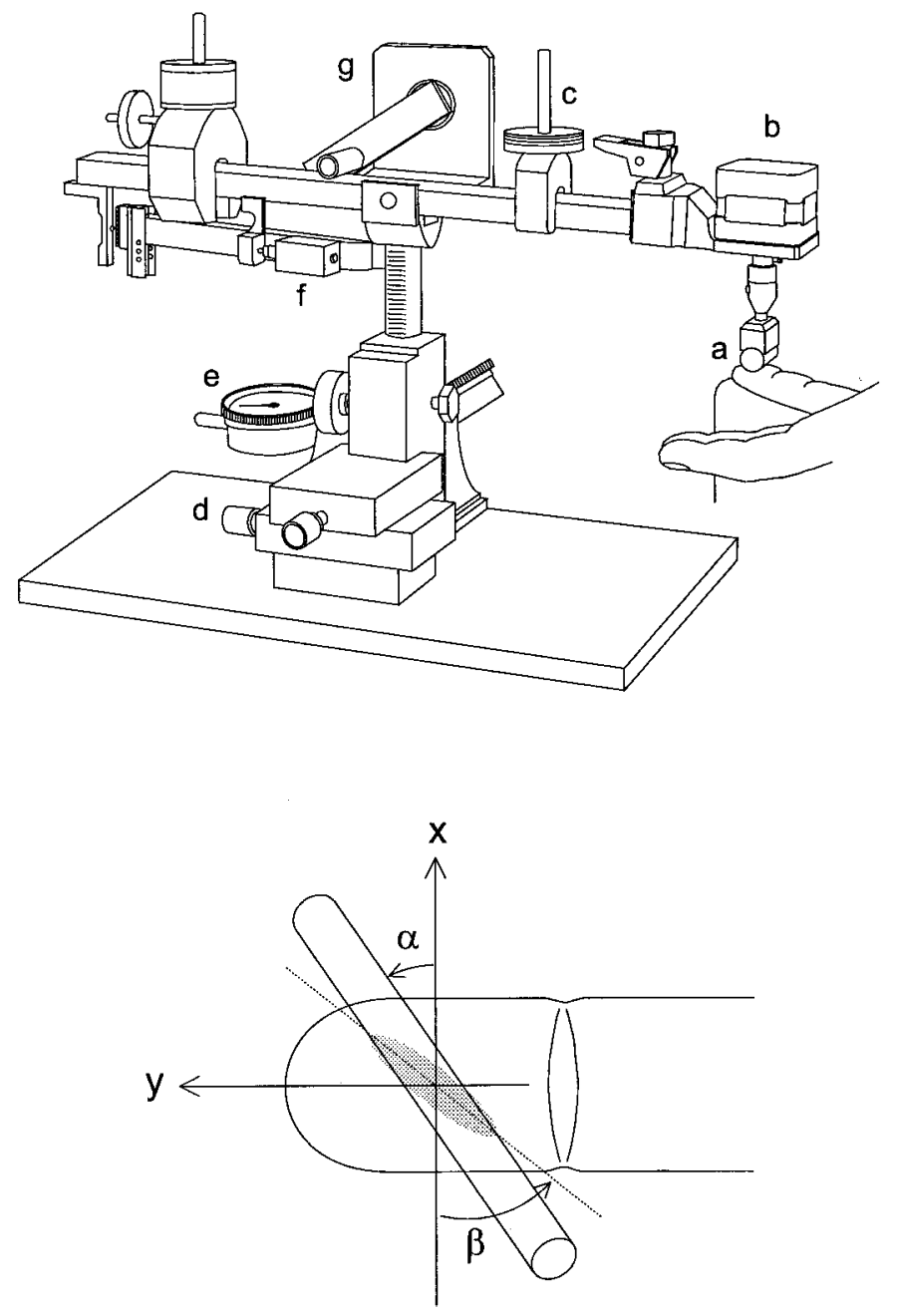

Figure 1. Balanced beam stimulator (top) and the coordinate conventions (bottom). A cylinder $(a)$ was mounted on the stepping motor $(b)$. Force was set by the weight $(c)$, and position was adjusted by the micrometers $(d)$ and dial indicators $(e)$. Activating relay $(f)$ released the beam, and damper $(g)$ ensured smooth motion. The $x$ - and $y$-axes passed through the receptive field center and were orthogonal to and parallel to the axis of the finger, respectively. The orientation of the cylinder was defined by angle $\alpha$ (positive, counterclockwise), and the orientation of the contact ellipse was defined by angle $\beta$.

described the responses of SAIs to cylinders of different curvature applied to the receptive field center, but there has been no analysis of cutaneous afferent population responses for cylindrical stimuli and no study of the representation of the orientation of the cylinder in the neural responses.

In this paper we measure the ability of humans to discriminate the orientation of cylinders passively contacting the fingerpad. In matching single-fiber recordings in anesthetized monkeys, we characterize the responses of the population of SAIs, showing how the orientation of the stimulus is represented in the neural responses, and we relate these to the human performance.

\section{MATERIALS AND METHODS}

Stimulus. The set of stimuli consisted of seven Delrin cylinders with radii of $1.44,1.92,2.94,3.90,5.81$, and $12.4 \mathrm{~mm}$ or $\infty$ (flat); the corresponding curvatures, given by the reciprocal of the radii, were 694, 521, 340, 256, $172,80.6$, and $0 \mathrm{~m}^{-1}$, respectively. The cylinders were attached to a stepping motor, which was mounted on the balanced beam of the stimulator (Fig. 1, top). When a relay was triggered, the beam was released, and the cylinder was lowered to contact the fingerpad. Static contact force was set by counterweights, and a damper ensured smooth motion with a velocity at contact of $20 \mathrm{~mm} \cdot \mathrm{sec}^{-1}$. The orientation of the cylinder $\alpha$ could be adjusted rapidly under computer control with a resolution of $0.9^{\circ} /$ step (Fig. 1, bottom). A pair of $x-y$ micrometers and dial indicators, attached to the stimulator, allowed the position at which the cylinders contacted the skin to be adjusted with a resolution of $0.01 \mathrm{~mm}$.

Human psychophysics. Six healthy humans (four male and two female) ranging in age from 22 to 48 years volunteered for these experiments, which were approved by the University of Melbourne Human Ethics Committee. The subject was seated comfortably with the forearm supinated, and the index finger of the dominant hand was anchored in a bed of Plasticine. A curtain prevented the subject from seeing either the stimulator or his or her finger. The stimulator was positioned with the surfaces of the cylinders located $1 \mathrm{~mm}$ above the skin. At contact, the line of action of the force was orthogonal to the plane tangential to the fingerpad. Contact force was set at $40 \mathrm{gm}$ weight $(0.392 \mathrm{~N})$. This force was chosen to allow comparison with previous tactile studies of shaped stimuli (e.g., Srinivasan and LaMotte, 1987; Goodwin et al., 1991); it is at the low end of forces generally used by humans in manipulative tasks (Johansson, 1996) and is particularly pertinent to "delicate" manipulations such as writing with a pen. In the first series of experiments, the subject's ability to discriminate the orientation $\alpha$ of the cylinder with curvature of $521 \mathrm{~m}^{-1}$ (radius, $1.92 \mathrm{~mm}$ ) was measured using a forced choice paradigm. A trial consisted of a pair of stimuli. For the first stimulus in the pair (the standard) the axis of the cylinder was at right angles to the axis of the finger $(\alpha=0)$. In some trials the second or comparison stimulus was at the same orientation as the standard $\left(S_{\mathrm{s}}\right)$, and for other trials the comparison stimulus was at a different orientation $\left(S_{\mathrm{d}}\right)$; the change in orientation was in a clockwise direction so that $\alpha$ was negative. The subject's task was to respond that both stimuli in the pair were the same $\left(R_{\mathrm{s}}\right)$ or that they were different $\left(R_{\mathrm{d}}\right)$. On each day five blocks of trials were run with the comparison stimulus having an orientation of $-1.8,-3.6,-5.4,-9$, or $-13.5^{\circ}$, respectively. Each block consisted of 20 trials with $10 S_{\mathrm{s}}$ and $10 S_{\mathrm{d}}$ pairs distributed randomly. The order of presentation of the blocks was varied randomly from day to day and from subject to subject. Data were collected for $10 \mathrm{~d}$ (200 trials at each orientation). To avoid auditory cues from the stepping motor, it was always advanced before presentation of either the standard or the comparison stimulus. For the standard stimuli and the $S_{\mathrm{s}}$ comparison stimuli, the stepping motor was advanced $180^{\circ}$; for the $S_{\mathrm{d}}$ comparison stimuli it was advanced $180^{\circ}$ plus the desired difference. The stimulus was in contact with the skin for $1 \mathrm{sec}$, and the interval between the standard and comparison stimuli in a trial was $\sim 1.5 \mathrm{sec}$. The point of contact of each stimulus was varied randomly along the axis of the finger over a range of $\sim 2 \mathrm{~mm}$. From the conditional probabilities, $P\left(R_{\mathrm{d}} / S_{\mathrm{d}}\right)$ and $P\left(R_{\mathrm{d}} / S_{\mathrm{s}}\right)$, the bias-free measure $d^{\prime}$ was calculated (Johnson, 1980).

A second series of experiments was conducted, which was identical to the first series except that the curvature of the cylinder was $172 \mathrm{~m}^{-1}$ (radius, $5.81 \mathrm{~mm}$ ), and there were six orientations, which had values of $-0.9,-1.8,-3.6,-4.5,-7.2$, and $-9^{\circ}$ respectively.

Neural recording. Experiments, approved by the University of Melbourne Animal Ethics Committee, were performed on five Macaca nemestrina monkeys weighing from 2.0 to $8.2 \mathrm{~kg}$. After a dose of ketamine hydrochloride ( $15 \mathrm{mg} / \mathrm{kg}$, i.m.) plus atropine sulfate $(60 \mu \mathrm{g} / \mathrm{kg}$, i.m.), an endotracheal tube and an intraperitoneal catheter were inserted. Surgical anesthesia was induced by administration of sodium pentobarbitone $(15-20 \mathrm{mg} / \mathrm{kg}$, i.v.) and was maintained throughout the experiment by sodium pentobarbitone diluted in saline $(15 \mathrm{mg} / \mathrm{ml}$, i.p.) administered hourly through the catheter (dose as required). The catheter was also used for fluid replacement. Body temperature was monitored by a rectal thermometer and was maintained at $37^{\circ} \mathrm{C}$ by a heating blanket. Respiration rate, end-tidal carbon dioxide level, blood pressure, heart rate, and oxygen saturation levels were monitored throughout the experiment. Antibiotic cover was provided by amoxycillin $(18 \mathrm{mg} / \mathrm{kg}$, i.m.) every $6 \mathrm{hr}$ and, at the end of the experiment, by a dose of $50 \mathrm{mg} / \mathrm{kg}$ benzathine penicillin, $30 \mathrm{mg} / \mathrm{kg}$ procaine penicillin, and $19 \mathrm{mg} / \mathrm{kg}$ benzylpenicillin. In four successive experiments on a monkey the median nerve was exposed at four sites, and single fibers were isolated by microdissection. The first two dissections were in the upper arms, and the last two were in the lower arms; at least 2 weeks elapsed between experiments. At the end of each experiment, the dissection was sutured in layers, and the monkey was returned to a heated, padded cage to recover.

Isolated action potentials recorded from a platinum hook electrode were amplified, stored on a digital oscilloscope, and passed through a 
A. Curvature $521 \mathrm{~m}^{-1}$

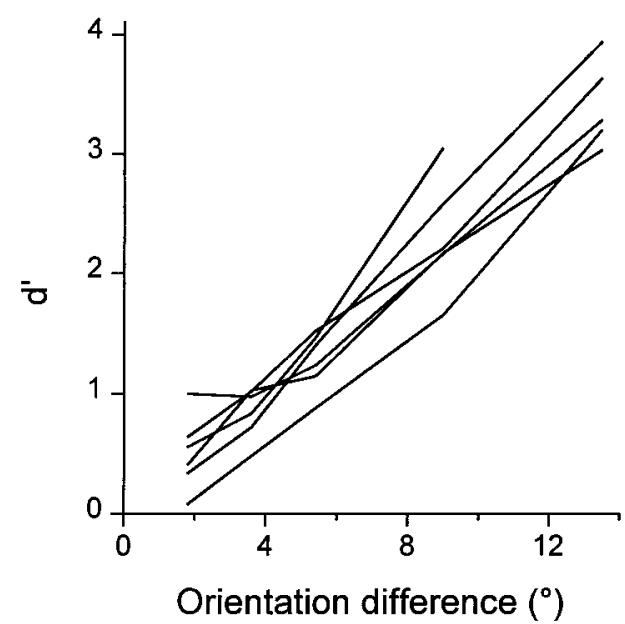

B. Curvature $172 \mathrm{~m}^{-1}$

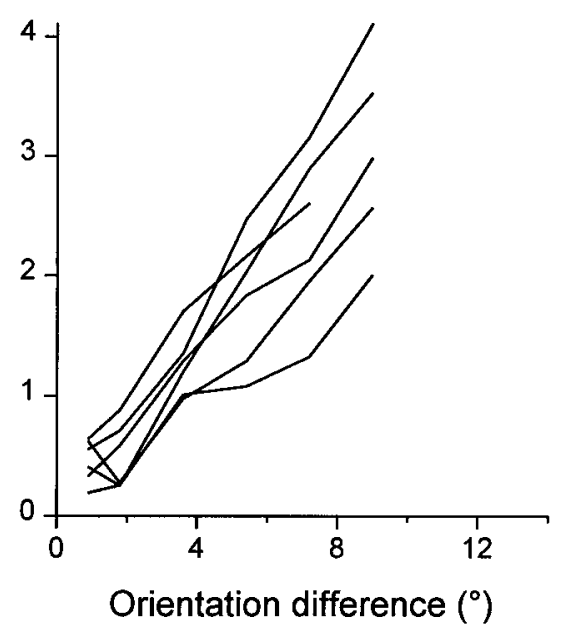

C. Subjects combined

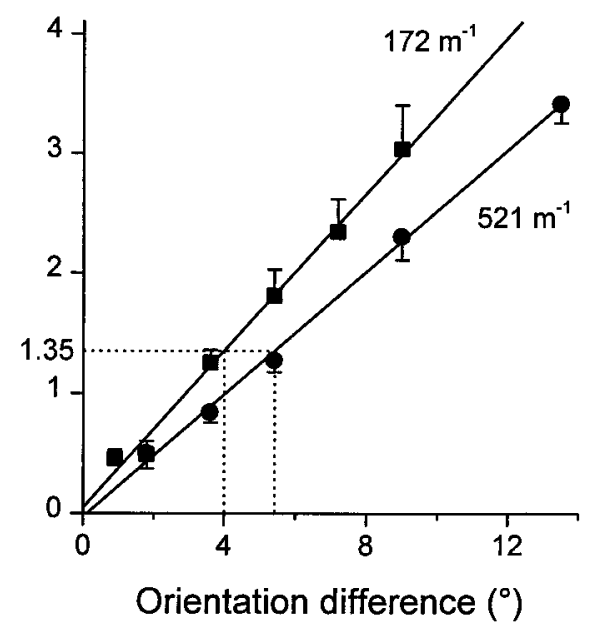

Figure 2. Human discrimination of the orientation of a cylinder contacting the fingerpad. $A$, Cylinder with curvature of $521 \mathrm{~m}^{-1}$ (radius, $1.92 \mathrm{~mm}$ ). For five curves (5 subjects) there are five data points at orientation differences of 1.8, 3.6, 5.4, 9, and $13.5^{\circ}$. For one subject the data point at $13.5^{\circ}$ is not shown, because $\mathrm{d}^{\prime}>4$ is not meaningful (Macmillan and Creelman, 1991). At each data point there are 200 observations. B, Same six subjects for the cylinder with curvature of $172 \mathrm{~m}^{-1}$ (radius, $5.81 \mathrm{~mm}$ ). For each curve (subject) there are six data points at orientation differences of $0.9,1.8,3.6,5.4,7.2$, and $9^{\circ}$; at each data point there are 200 observations $C$, Data points show means and unilateral SEMs for psychometric functions averaged over the six subjects. Lines show linear regression of the data points.

time-amplitude window discriminator. These signals were sampled at 10 $\mathrm{kHz}(0.1 \mathrm{msec}$ resolution) and stored on disk for off-line analysis. Single fibers were classified as SAIs, fast-adapting type I afferents (FAIs), or FAIIs by the established criteria of responses to static stimuli, responses to rapidly changing stimuli, and receptive field size (Vallbo and Johansson, 1984). Initially, receptive field positions were estimated using von Frey hairs. Only SAIs were studied and only if the receptive field was located on the central region of a fingerpad. The finger innervated by the isolated afferent was immobilized in modeling clay with the nail secured by cotton thread. The center of the receptive field was then determined more precisely as follows. A sphere with radius of $1.44 \mathrm{~mm}$ (curvature, $694 \mathrm{~m}^{-1}$ ) was attached to the stimulator and applied, with a contact force of $15 \mathrm{gm}$ weight $(0.147 \mathrm{~N})$, to points separated by $0.5 \mathrm{~mm}$ along two orthogonal axes through the estimated receptive field center; one axis was parallel to the axis of the finger, and the other was at right angles to it. At each point the total response over $1 \mathrm{sec}$ was measured, and the point of maximum response was determined. The process was repeated successively around this point to establish the center of the receptive field.

Receptive field response profiles were measured for the seven cylinders with the axis of the cylinder orthogonal to the axis of the finger $(\alpha$ $\left.=0^{\circ}\right)$. One of the cylinders was applied to the receptive field at points separated by $0.5 \mathrm{~mm}$ along the $y$-axis (Fig. 1). The surface of the cylinder was positioned $0.5 \mathrm{~mm}$ above the skin, and the line of action of the force was orthogonal to the plane tangential to the fingerpad at the receptive field center. Contact force was set at $15 \mathrm{gm}$ weight $(0.147 \mathrm{~N})$. At each point the stimulus was applied seven times, contacting the finger for 1.5 $\mathrm{sec}$ and then raised off the finger for $2.5 \mathrm{sec}$. For analysis, the first application was ignored, and responses for the last six applications (each preceded by the same stimulus with the same interstimulus interval) were used. The process was repeated for the remaining six cylinders with the order of presentation of the seven cylinders varied randomly from afferent to afferent. Responses were measured by the number of action potentials occurring during the first second of response.

For the two cylinders used in the human psychophysics experiments (curvatures, 521 and $172 \mathrm{~m}^{-1}$ ), response profiles were measured in both spatial dimensions on the skin at five different orientations as follows. One of the cylinders was positioned at points along the $y$-axis separated by $0.5 \mathrm{~mm}$. At each point the stimulus was presented in a block of 31 trials. The first trial was ignored in the analysis, and the remaining 30 trials consisted of six presentations at each of the five orientations at 0 , \pm 4.5 , and $\pm 9^{\circ}$. The order of the 30 trials was randomized. This process was then repeated along lines parallel to the axis of the finger at $x= \pm 1$, \pm 2 , and $3 \mathrm{~mm}$. The entire process was then executed for the second cylinder. The order in which the two cylinders were selected was randomized from afferent to afferent.

To define the profiles as completely as possible, for each afferent we examined the maximum number of points located on the relatively flat central portion of the fingerpad. The proximal limit was determined by the interphalangeal crease, the distal limit by the curvature of the fingertip, and the medial and lateral limits by the curvature of the sides of the finger. A complete matrix took $\sim 7 \mathrm{hr}$.

The process of manufacturing the cylinders and their attachments to the stepping motor was such that there was a different small offset in the $y$ direction for each cylinder. These offsets were measured with a micrometer and were compensated for in the analysis.

\section{RESULTS}

\section{Human psychophysics}

When a cylinder of curvature of $521 \mathrm{~m}^{-1}$ contacted the fingerpad, humans were able to discriminate small differences in the orientation of the cylinder. In Figure $2 A$ the index of discriminability $d^{\prime}$ is plotted as a function of the difference in orientation between the standard and comparison stimuli. The psychometric function was similar for all six subjects, with $d^{\prime}$ increasing approximately linearly with an increase in the orientation difference. For each subject the difference limen was calculated as follows. Regression of the five data points yielded a straight line from which the orientation difference corresponding to a $d^{\prime}$ value of 1.35 could be obtained; this is equivalent to a difference in orientation that could be discriminated with a probability of $75 \%$. Table 1 shows these difference limens. Similar behavior occurred for the cylinder with a curvature of $172 \mathrm{~m}^{-1}$. Comparison of discrimination for the two cylinders is seen clearly in Figure $2 C$, which shows the psychometric functions averaged over the six subjects for each of the cylinders. At all orientation differences, discrimination was more acute for the less curved stimulus. For each subject, the difference limen shown in Table 1 was less for the cylinder with curvature of $172 \mathrm{~m}^{-1}$ than for the cylinder with curvature of 521 $\mathrm{m}^{-1}$ (one-tailed paired $t$ test, $p=0.001$ ). Because the position of 


\begin{tabular}{lll}
\hline \multicolumn{2}{l}{ Table 1. Difference limens for cylinder orientation } \\
Subject & $521 \mathrm{~m}^{-1}$ & $172 \mathrm{~m}^{-1}$ \\
\hline S1 & 4.84 & 3.01 \\
S2 & 5.74 & 4.90 \\
S3 & 7.01 & 6.41 \\
S4 & 4.86 & 3.09 \\
S5 & 4.56 & 3.77 \\
S6 & 5.25 & 4.10 \\
Mean & 5.38 & 4.21 \\
SEM & 0.367 & 0.523 \\
\hline
\end{tabular}

The difference limens for a cylinder with curvature of $172 \mathrm{~m}^{-1}$ were significantly smaller than those for a cylinder with curvature of $521 \mathrm{~m}^{-1}$ (paired $t$ test: one-tailed, $p=0.001 ;$ two-tailed, $p=0.003$ ).

contact of the cylinders on the skin was varied randomly, the discrimination could not have been based on spurious simple cues such as a shift in position of a single end point of the contact area.

\section{Receptive field profiles}

Responses of 13 SAIs were measured when cylinders, with their axes orthogonal to the axis of the finger $\left(\alpha=0^{\circ}\right.$ in Fig. 1$)$, were placed at various positions in the receptive field. For such a cylinder, a change of position in the $x$ direction has no effect on the geometry of contact and thus has no effect on the response of the afferent. Receptive fields were characterized by measuring the responses when the cylinder was positioned at points separated by $0.5 \mathrm{~mm}$ along the $y$-axis. The numbers of impulses elicited during the first second of response are shown for a typical SAI in Figure $3 A$. The small SEs verify the consistency of the responses. For each cylinder, the response profile peaked when the cylinder was close to the receptive field center and decreased as the cylinder was placed more peripherally in the receptive field. An increase in the curvature of the cylinder had two effects. The peak value of the profile increased, and the width of the profile decreased.

All 13 SAIs behaved in the same way, except that some afferents were more sensitive than others. Thus, for the same stimulus, some afferents had a greater response than others. The effect of the sensitivity of an individual fiber was eliminated by normalizing; the responses were divided by an average response for that fiber. The normalization factor is a measure of the sensitivity of the afferent. The most responsive afferent was 5.1 times as sensitive as the least responsive afferent, and the 13 normalization factors were distributed approximately normally, with a coefficient of variation of 0.38 , a result similar to that found by Goodwin et al. (1995) for spheres. The normalized profiles, illustrated in Figure $3 B$ for the cylinder with a curvature of $340 \mathrm{~m}^{-1}$, were consistent from fiber to fiber, indicating that, apart from the sensitivity factor, all SAIs had the same underlying stimulusresponse characteristics.

Normalized response profiles pooled for the 13 afferents for all seven cylinders are shown in Figure $4 A$. It can be seen clearly that as the curvature of the cylinder increased, the profile peak increased, and the profile width decreased. There was a small proximal shift in the position of the peak with a decrease in the curvature of the cylinder, probably related to the curvature of the finger. In this case the small SEs indicate the consistency of the underlying profiles from fiber to fiber. For further analysis, which is described later in the paper, it is useful to define these profiles mathematically. The profile for each cylinder fits closely to the function $a e^{-b y^{2}}$. Table 2 gives the values of the constants $a$ and $b$, which were determined by nonlinear regression using the Levenberg-Marquardt algorithm (Press et al., 1986). The constant $a$ (the height of the profile) indicates the dependence on local curvature of the Merkel complexes in conjunction with skin mechanics. As seen in Figure $4 B$, for low curvatures responses increased rapidly with increasing curvature and then started to saturate for higher curvatures. A commonly used measure of the extent of profiles such as those in Figure $4 A$ is their width at half-height. From the equation above, the width at half-height is given by $w=1.67 / \sqrt{ } b$. This parameter, shown in Figure $4 B$, indicates how the "size" of the receptive field decreased as the curvature of the cylinder increased. For comparison, equivalents of the constants $a$ and $w$ for spherical stimuli, measured by Goodwin et al. (1995), are shown by the broken lines.

For any stimulus, the receptive field profile of an afferent is defined by the responses at points in the two-dimensional plane of the receptive field. For a cylinder, the geometry of which is invariant along its axis, these profiles are effectively onedimensional, as illustrated in Figure 5. Such profiles can be interpreted as the responses of the population of afferents from a fingerpad in contact with the cylinder, with the simplifying assumption that all afferents have the same sensitivity.

\section{Change in orientation}

If the finger were perfectly flat, and the skin mechanics were isotropic, then changing the orientation of the cylinder with respect to the finger would simply result in a rotation of the response profile through that same angle. Thus, for example, changing the orientation of the cylinder with curvature of 521 $\mathrm{m}^{-1}$ by $9^{\circ}$ would result in the profile shown in Figure 5, right panel, rotating by $9^{\circ}$ about the $z$ (normalized response) axis. For a cylinder at an orientation $\alpha$, the resulting response profile would be given by $a e^{-b u^{2}}$, where $u=y \cos \alpha-x \sin \alpha$. However, the finger is not flat, and the analysis of Fearing and Binford (1991) for a rigid cylinder contacting an elastic cylinder suggests that the rotation of the population response $(\theta)$ may not match the rotation of the cylinder $(\alpha)$ exactly. Thus we measured directly the nature and extent of rotation in the population response.

Responses at a matrix of points separated by $0.5 \mathrm{~mm}$ in the $y$ direction and $1 \mathrm{~mm}$ in the $x$ direction were measured for 11 SAIs using the cylinder with curvature of $172 \mathrm{~m}^{-1}$ and for 10 SAIs using the cylinder with curvature of $521 \mathrm{~m}^{-1}$. As before, all the afferents behaved in the same way, differing only by a scaling factor proportional to their sensitivity. Thus, the normalized responses could be combined to show the underlying rotated profiles common to all afferents. There are two complementary ways of visualizing rotation in the population response. In Figure 6 , profiles varying in one spatial dimension $(y)$ are shown along lines parallel to the axis of the finger. Three characteristics can be seen. First, at $x=0$ (Fig. $6 B$ ) the profiles at all five orientations are coincident. Second, along a line away from the origin (Fig. $6 C)$ the profile shifts with rotation, and the shift is greater for greater rotations. Third, along a line on the opposite side of the origin (Fig. 6A) the profile shifts in the opposite direction. In these figures it is easy to gauge the magnitude of the shifts, but it is not so easy to visualize the rotation of the profile in both spatial dimensions. This is more easily seen in Figure 7, which shows the rotation clearly for both spatial dimensions; the disadvantage here is that it is not easy to assess small quantitative changes in three-dimensional plots.

Figures 6 and 7 demonstrate a rotation of the response profile. The extent of the rotation $(\theta)$ was estimated by nonlinear regres- 


\section{A. Single SAI}

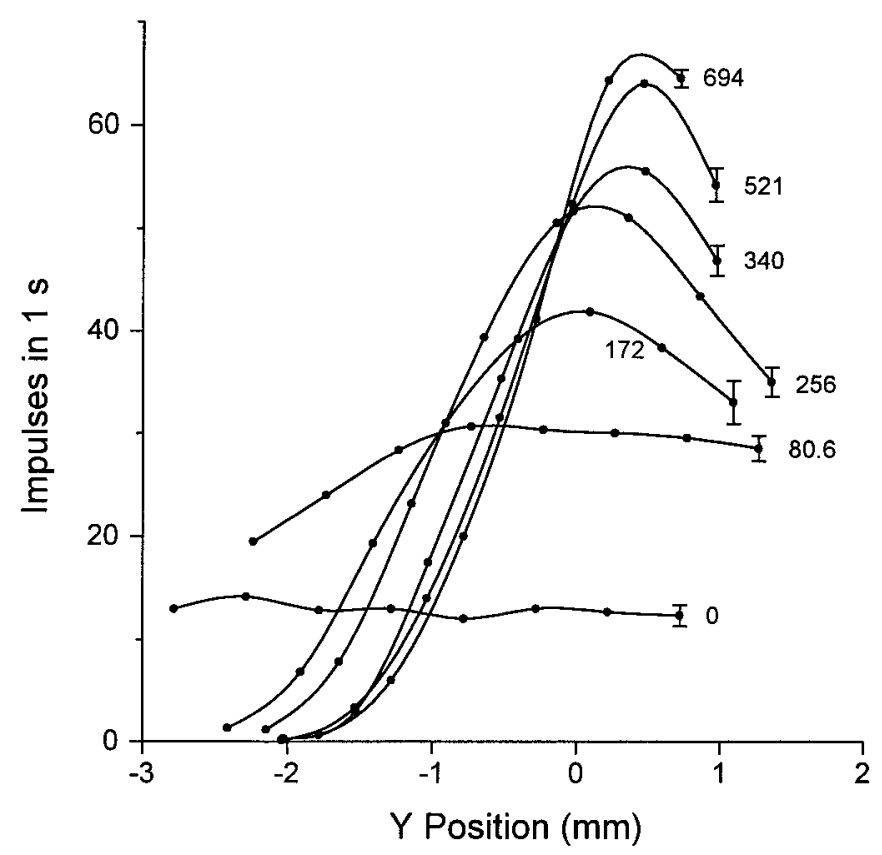

B. 13 afferents, curvature $340 \mathrm{~m}^{-1}$

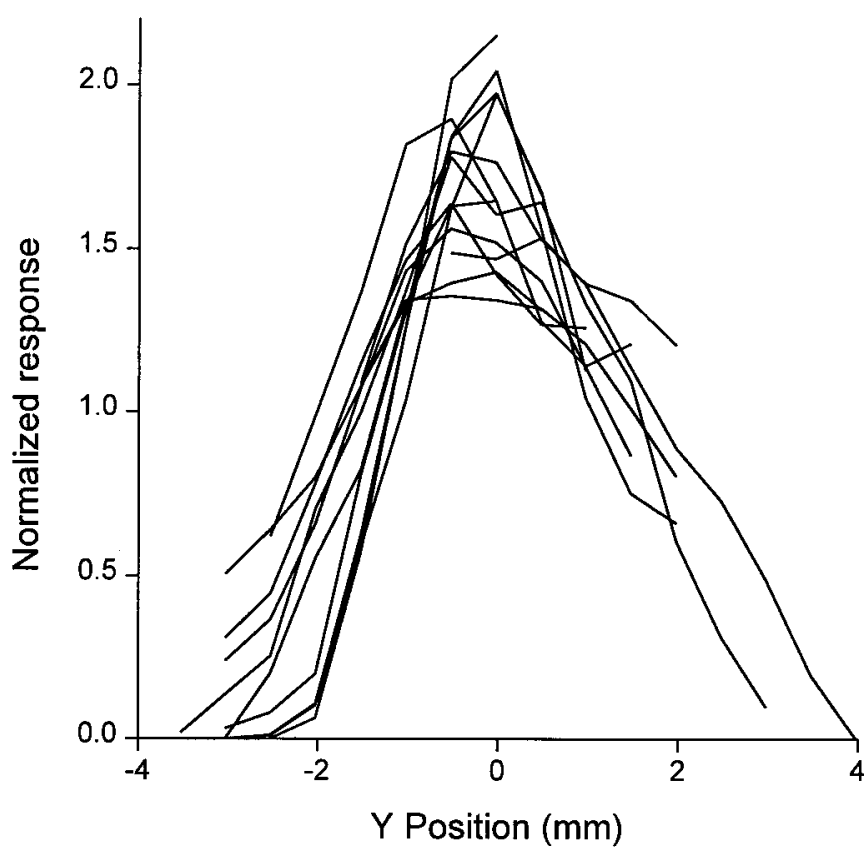

Figure 3. Response profiles for cylinders with their axes orthogonal to the axis of the finger. $A$, Responses of a single SAI (mean \pm SEM, $n=6$ ) to the seven cylinders positioned at points separated by $0.5 \mathrm{~mm}$ along the $y$-axis. Numbers indicate the curvature of the cylinder in $\mathrm{m}^{-1}$. For clarity, SEMs are only shown at one point for each line, but they are similar at all the other points. $B$, Normalized profiles for 13 SAIs responding to the cylinder with curvature of $340 \mathrm{~m}^{-1}$. The normalizing factor was the average response over the 49 points that were common to all 13 afferents $(7$ cylinders by 7 positions about the center).

sion, fitting the rotated profiles to the function $a e^{-b(y \cos \theta-x \sin \theta)^{2}}$. As seen in Table 3, the orientation of the SAI response profile was not the same as the orientation of the cylinder. In all cases the magnitude of the response orientation was greater than the magnitude of the cylinder orientation. Furthermore, the difference in angle $(\theta-\alpha)$ increased with increasing cylinder orientation, and the magnitude of the difference was greater for the less curved cylinder (curvature, $172 \mathrm{~m}^{-1}$ ) than for the more curved cylinder (curvature, $521 \mathrm{~m}^{-1}$ ).

\section{Orientation of contact areas}

When a rigid cylinder contacts a finger, which is a curved, compliant body, the geometry of the contact is complex and depends on the curvature of the cylinder and on the angle of the cylinder with respect to the axis of the finger. An idea of the likely nature of this contact can be obtained from the analysis by Fearing and Binford (1991) for the contact between a rigid cylinder and a cylindrical elastic "finger." They showed that the contact area was elliptical and that the orientation of the major axis of the ellipse (Fig. $1, \beta$ ) was related to the orientation $\alpha$ of the cylinder by the expression:

$$
\tan 2 \beta=\frac{\kappa_{\mathrm{c}} \sin 2 \alpha}{\kappa_{\mathrm{c}} \cos 2 \alpha-\kappa_{\mathrm{f}}}
$$

where $\kappa_{\mathrm{c}}$ and $\kappa_{\mathrm{f}}$ are the curvature of the cylinder and the elastic finger, respectively. The difference between the two orientations $(\beta-\alpha)$ is shown in Figure 8 as a function of the orientation $\alpha$ for the two cylinders with curvature of 521 and $172 \mathrm{~m}^{-1}$ for a number of elastic fingers of different curvature. The magnitude of the orientation of the contact ellipse is greater than the magnitude of the orientation of the cylinder, and the difference between them increases as the cylinder is angled closer toward the axis of the finger (closer to $\alpha= \pm 90^{\circ}$ ). The difference also increases as the finger becomes more curved, and the difference is greater for the less curved cylin$\operatorname{der}\left(172 \mathrm{~m}^{-1}\right)$ than for the more curved cylinder $\left(521 \mathrm{~m}^{-1}\right)$.

We estimated the contact areas in our human subjects by inking the cylinders and applying them to the fingerpads at angles of 0 , -4.5 , and $-8.1^{\circ}$ using the same stimulator and stimulus conditions as used in the psychophysics experiments. A set of fingerprints from a typical subject is shown in Figure $8 C$. The contact areas were elliptical, and the orientation of the major axis (estimated by eye) averaged over the six subjects was -6.0 and $-9.6^{\circ}$ for the cylinder with curvature of $521 \mathrm{~m}^{-1}$ at orientations of -4.5 and $-8.1^{\circ}$, respectively and -6.9 and $-12.3^{\circ}$ for the cylinder with curvature of $172 \mathrm{~m}^{-1}$ at orientations of -4.5 and $-8.1^{\circ}$, respectively. These results are shown by crosses in Figure 8 . These measurements are approximate only, and there are only two angles and two cylinders, but they show similar characteristics to the results of Fearing and Binford (1991): the magnitude of the difference in orientation was greater for the less curved cylinder, and the difference increased with orientation for the less curved cylinder but did not change with orientation for the more curved cylinder.

When the cylinders were applied to monkeys' fingers, we measured the orientation of the SAI response profiles directly. The differences between the orientation of the response profile and the orientation of the cylinder $(\theta-\alpha$ in Table 3$)$ are plotted as open squares in Figure 8 . This difference also increased with 


\section{A. 13 afferents combined}

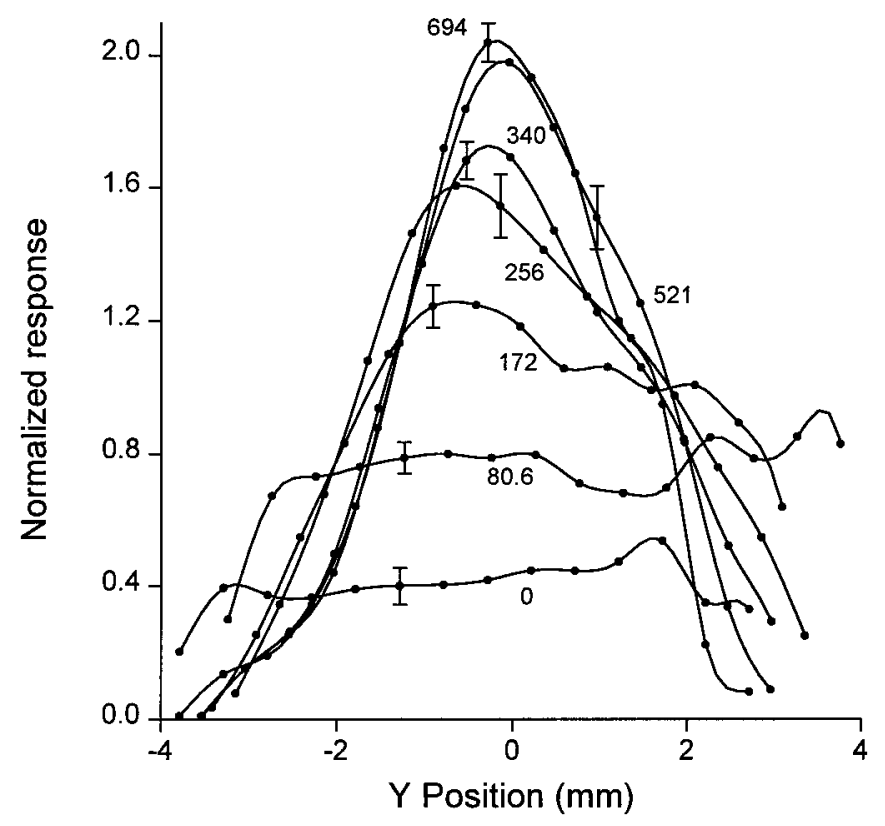

\section{B. Constants from regression}

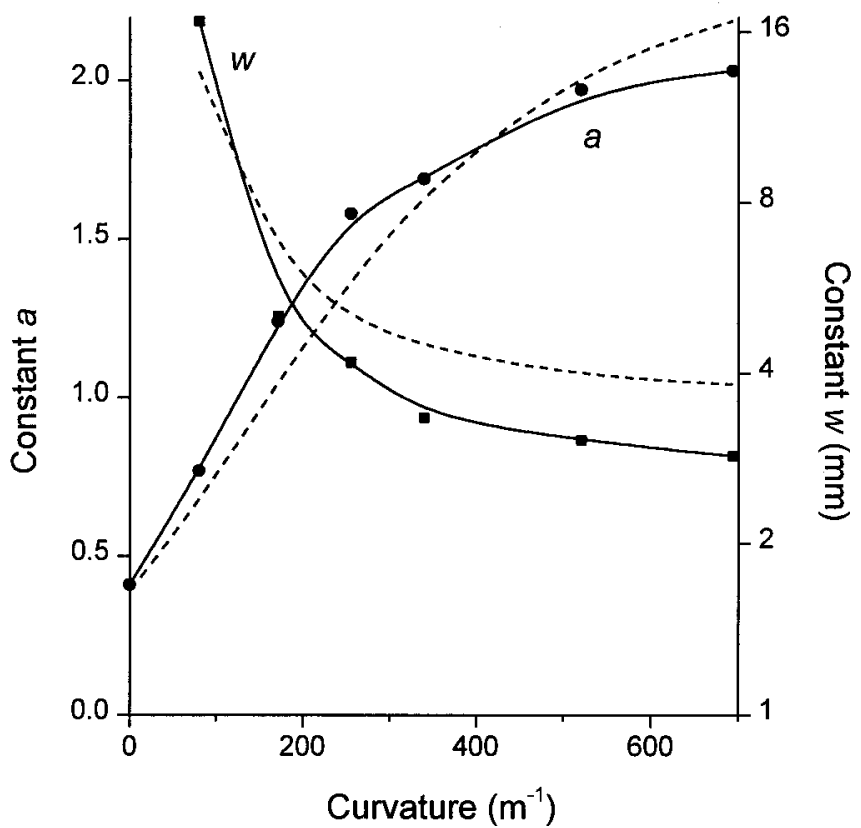

Figure 4. Profiles for the pool of SAIs. A, Normalized profiles (mean \pm SEM) for the 13 afferents responding to all seven cylinders. Numbers indicate the curvature of the cylinder in $\mathrm{m}^{-1}$. Because the profile could not be measured over the full extent from -3.5 to $3.5 \mathrm{~mm}$ for each afferent, the number of data points is different for different positions; $n=13$ for the more central points and decreases for more peripheral points. Nevertheless, the single SEM shown for each line is similar for all points on the line. $B$, Data points and solid, splined lines show the height of the receptive field profile $(a)$ and its width at half-height $(w)$ for all seven cylinders. Broken lines show equivalent values of the constants $a$ and $w$ for spherical surfaces from the data of Goodwin et al. (1995).

\begin{tabular}{lllllr}
\hline \multicolumn{6}{l}{ Table 2. Regression of response profiles } \\
$\begin{array}{l}\text { Curvature } \\
\left(\mathrm{m}^{-1}\right)\end{array}$ & $a$ & $\begin{array}{l}b \\
\left(\mathrm{~mm}^{-2}\right)\end{array}$ & $\begin{array}{l}w \\
(\mathrm{~mm})\end{array}$ & $n$ & \multicolumn{1}{c}{$F$} \\
\hline 0 & 0.41 & 0 & $\infty$ & 109 & 566 \\
80.6 & 0.77 & 0.01 & 16.7 & 120 & 779 \\
172 & 1.24 & 0.11 & 5.04 & 115 & 664 \\
256 & 1.58 & 0.16 & 4.18 & 116 & 1005 \\
340 & 1.69 & 0.25 & 3.34 & 116 & 1468 \\
521 & 1.97 & 0.30 & 3.05 & 116 & 1504 \\
694 & 2.03 & 0.34 & 2.86 & 117 & 1052 \\
\hline
\end{tabular}

Constants $a$ and $b$ were determined by least squares fit of the equation $a e^{-b y^{2}}$ to the SAI response profiles for the seven cylinders. $F$ values were calculated by dividing the regression mean square by the residual mean square; the high $F$ values indicate the close fit (all $p<<0.001 ; n$, number of data points. The width of the profile at half-height $w=1.67 / \mathrm{V} b$.

increasing orientation of the cylinder and was greater for the cylinder with the smaller curvature.

Human and monkey fingers are not cylindrical but have a higher curvature on the sides of the fingers and are flatter at the center of the fingerpad. Thus the effective curvature of the fingerpad is likely to be somewhere between flat $\left(0 \mathrm{~m}^{-1}\right)$ and the reciprocal of the radius of the finger $\left(\sim 100 \mathrm{~m}^{-1}\right.$ for humans and $\sim 250 \mathrm{~m}^{-1}$ for our monkeys). The crosses and squares in Figure 8 are consistent with this. Taken together, these results show that when cylinders contact the finger, the contact ellipse has a different orientation to the orientation of the cylinder, and the orientation of the SAI population response is correspondingly affected. The magnitude of the response orientation is greater than the magnitude of the cylinder orientation and is greater for less curved cylinders than for more curved cylinders, which may explain why the human difference limens were smaller for the less curved cylinder.

\section{DISCUSSION}

Humans are able to manipulate objects with a high degree of precision. The hand, which is unique to primates, relies on independent movements of the digits and on an opposable thumb (Tubiana, 1981). The accuracy of these hand movements depends on feedback of the parameters of the manipulated object. Such information is relayed by a number of classes of receptors, including joint receptors, muscle spindle afferents, and Golgi tendon organs (Gandevia et al., 1992). However, information about the local shape of a grasped object and its position of contact on the fingers can only be signaled by the cutaneous mechanoreceptive afferents (Goodwin, 1997). Cylinders represent an important class of relatively simple objects, the shape of which is completely defined by two parameters: the orientation of the axis of the cylinder, in which direction the curvature is zero, and the curvature in the orthogonal direction. We have investigated the representation of the curvature and orientation of a cylinder in the digital nerve discharge in monkeys and have related this to human psychophysical performance.

\section{Human performance: neural substrate}

When a highly curved cylinder (curvature, $521 \mathrm{~m}^{-1}$; radius, 1.92 $\mathrm{mm}$ ) was applied passively to the subjects' fingerpads, they were able to discriminate changes in orientation of $5.4^{\circ}$. For a less curved cylinder (curvature, $172 \mathrm{~m}^{-1}$; radius, $5.81 \mathrm{~mm}$ ), their 
Curvature $172 \mathrm{~m}^{-1}$

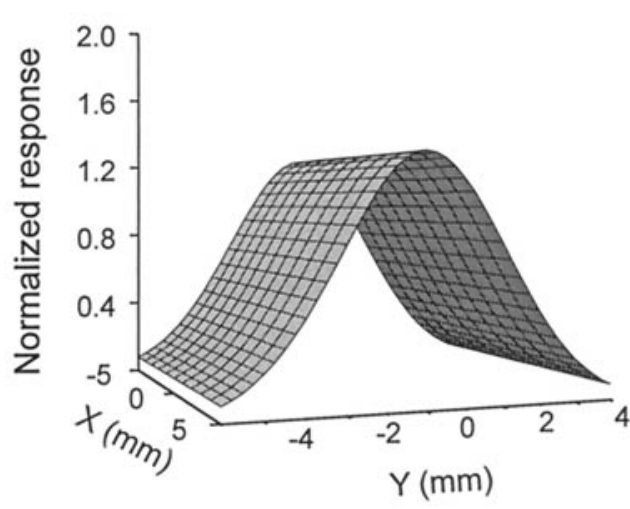

Curvature $340 \mathrm{~m}^{-1}$

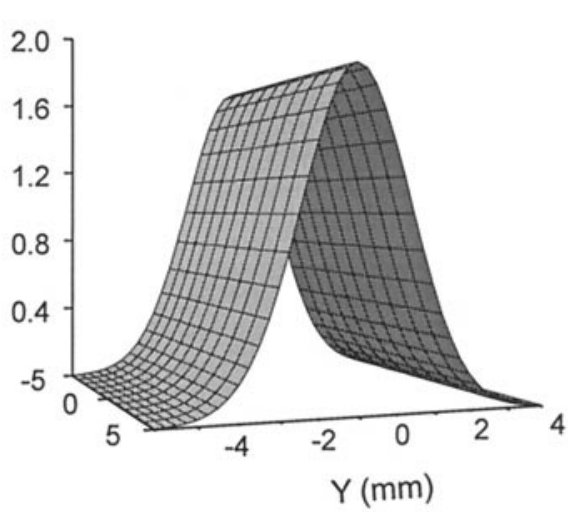

Curvature $521 \mathrm{~m}^{-1}$

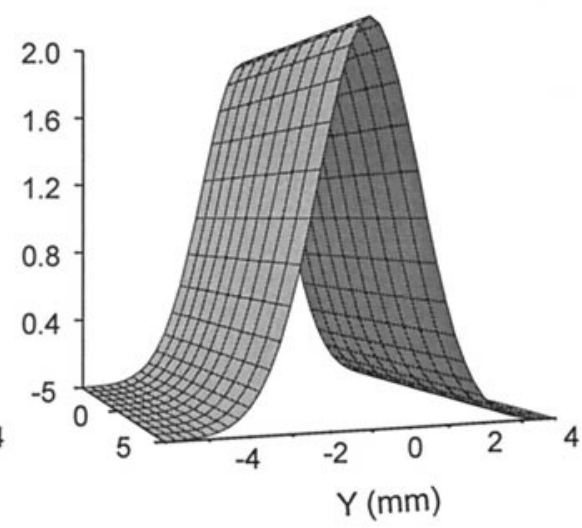

Figure 5. Population response profiles in both spatial dimensions illustrated for three of the cylinders. The axis of the cylinder was oriented along the $x$-axis $\left(\alpha=0^{\circ}\right)$. Profiles are plotted from the function $a e^{-b y^{2}}$. For curvatures of 172, 340, and $521 \mathrm{~m}^{-1}$, the constants $a$ and $b$ determined by regression are 1.24 and $0.11,1.69$ and 0.25 , and 1.97 and 0.30 , respectively.

A. $X=-1 \mathrm{~mm}$

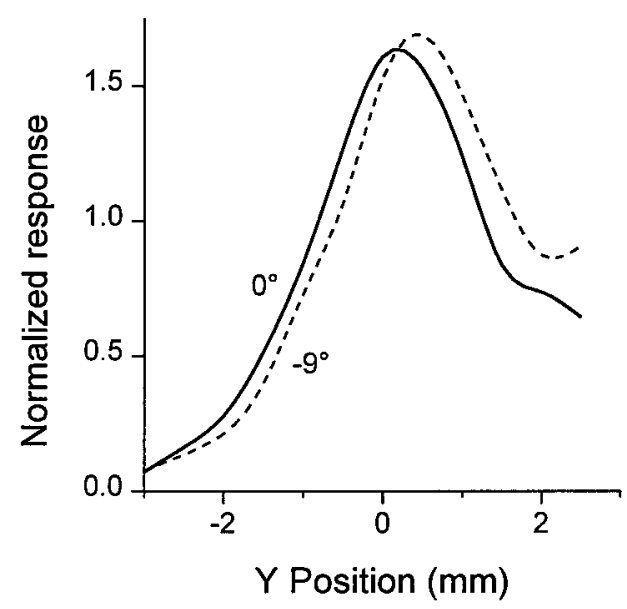

B. $X=0 \mathrm{~mm}$

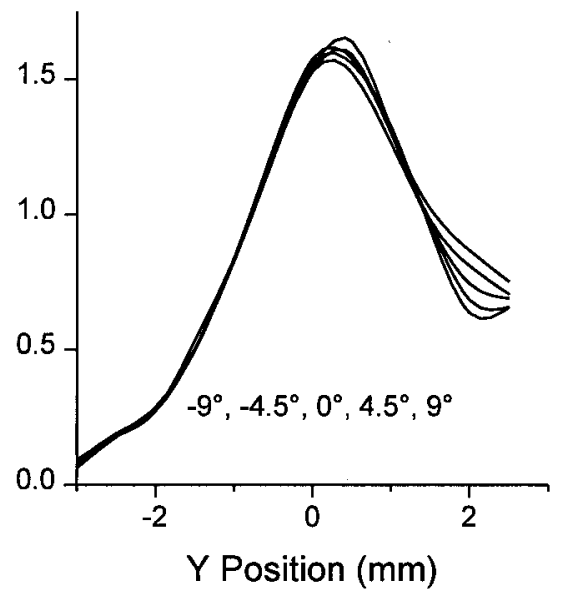

C. $X=3 \mathrm{~mm}$

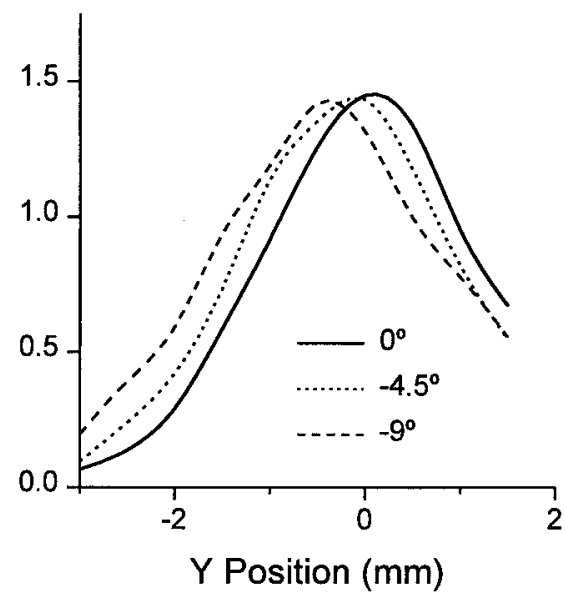

Figure 6. Normalized responses averaged over 10 SAIs responding to the cylinder with a curvature of $521 \mathrm{~m}^{-1}$. The $x$ - and $y$-axes were orthogonal to and parallel to the axis of the finger, respectively, and the orientation $\alpha$ of the cylinder (angle between its axis and the $x$-axis) was $0, \pm 4.5$, or $\pm 9^{\circ}$. For each profile the curve passes through data points separated by $0.5 \mathrm{~mm}$.

discrimination threshold of $4.2^{\circ}$ was significantly lower. These values are less than the $10^{\circ}$ difference limen found by Lechelt (1992) for a cylinder of curvature of $526 \mathrm{~m}^{-1}$ (radius, $1.9 \mathrm{~mm}$ ), probably because of cognitive factors resulting from the more complex paradigm of Lechelt (1992), which involved a comparison of four stimuli presented to both the left and right hands. Other tactile orientation data, such as the discrimination threshold of $14^{\circ}$ for a dot scanned over the finger at different orientations (Keyson and Houtsma, 1995), are not directly comparable.

In our experiments, the cylinders were applied passively to the skin with a constant static contact force and with an initial contact velocity of $20 \mathrm{~mm} \cdot \mathrm{sec}^{-1}$. In monkeys, only the SAIs responded appreciably to such stimuli. All SAIs had the same underlying stimulus-response characteristics, differing only by a scaling constant determined by the sensitivity of the afferent; thus it was possible to reconstruct the responses of a complete ideal SAI population to any of the cylinders at any orientation. For three parameters of the cylinder (curvature, position, and orientation), the only source of information relayed to the brain arises in the population of cutaneous afferents. The shape (curvature) of the cylinder is reflected in the shape of the SAI population response, which increases in magnitude and decreases in width for more curved cylinders. The position of contact on the skin is represented by the position of the response profile within the overall SAI population. For these two parameters of the stimulus, curvature and position, the results are analogous to our previous observation for spherical stimuli (Goodwin et al., 1995; Wheat et al., 1995). The dependence of the peak of the response profiles on curvature (Fig. 4, constant $a$ ) is similar to corresponding measurements made previously by LaMotte and Srinivasan (1993, 1996). Comparison of profile heights and widths (Fig. 4) for spheres, which have a single curvature in all directions, and cylinders, which have a single curvature in one direction and zero curvature in the orthogonal direction, should allow a refinement of existing skin mechanics models (Phillips and Johnson, 1981; Srinivasan, 1989; Srinivasan and Dandekar, 1996). LaMotte and 
A. Orientation $9^{\circ}$

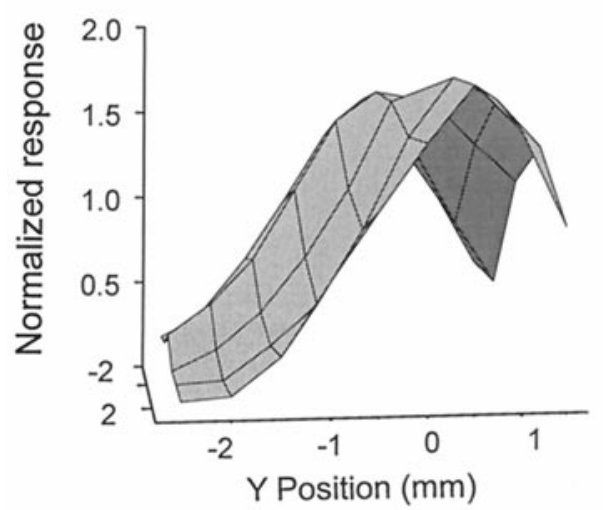

B. Orientation $0^{\circ}$

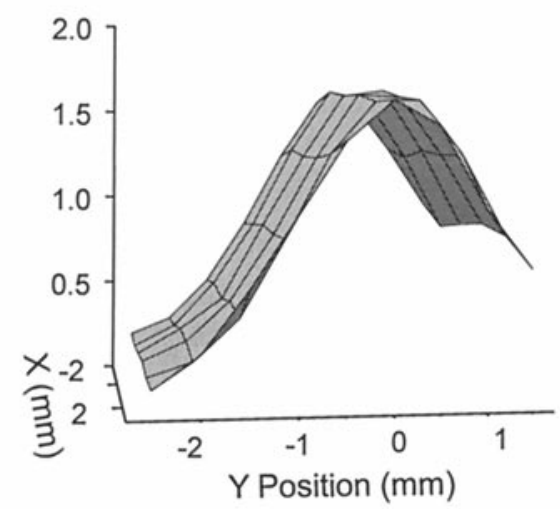

C. Orientation $-9^{\circ}$

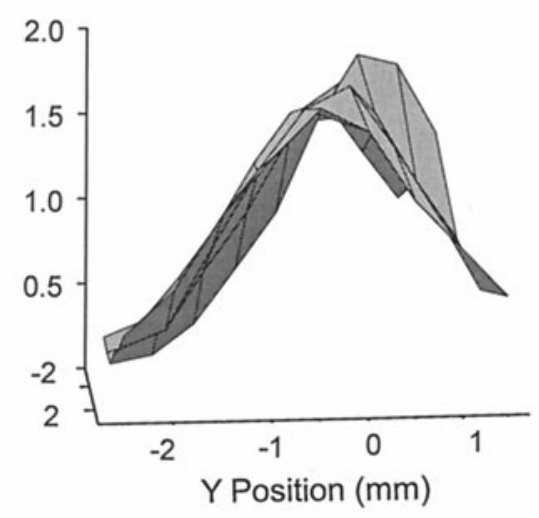

Figure 7. Normalized responses averaged over 10 SAIs responding to the cylinder with a curvature of $521 \mathrm{~m}^{-1}$. Profiles are shown for three orientations of the cylinder $\left(9,0\right.$, and $\left.-9^{\circ}\right)$. Data points are separated by $1 \mathrm{~mm}$ in the $x$ direction and by $0.5 \mathrm{~mm}$ in the $y$ direction.

\begin{tabular}{llll}
\hline \multicolumn{3}{l}{ Table 3. Rotation of response profiles } \\
$\begin{array}{cccc}\text { Cylinder } \\
\text { orientation }\left(\alpha^{\circ}\right)\end{array}$ & $\begin{array}{l}\text { Profile } \\
\text { orientation }\left(\theta^{\circ}\right)\end{array}$ & $\theta^{\circ}-\alpha^{\circ}$ & $F$ \\
\hline $\begin{array}{c}\text { Cylinder } 521 \mathrm{~m}^{-1} \\
9\end{array}$ & 11.3 & & \\
4.5 & 5.3 & 2.3 & 3134 \\
0 & -0.06 & 0.8 & 4234 \\
-4.5 & -6.13 & -0.06 & 4715 \\
-9 & -11.6 & -2.63 & 4288 \\
Cylinder $172 \mathrm{~m}^{-1}$ & & & 3965 \\
9 & 17.3 & 8.3 & \\
4.5 & 9.3 & 4.8 & 6042 \\
0 & 2.3 & 2.3 & 6747 \\
-4.5 & -6.0 & -1.5 & 5992 \\
-9 & -14.9 & -5.9 & 4724 \\
\end{tabular}

Cylinders with curvature of 521 or $172 \mathrm{~m}^{-1}$ were applied to the fingerpad at orientations $(\alpha)$ of $0, \pm 4.5$, and $\pm 9^{\circ}$. The rotation of the SAI response profile $\left(\theta^{\circ}\right)$ was calculated by least squares fit of the equation $a e^{-b(y \cos \theta-x \sin \theta)^{2}}$ using the Levenberg-Marquardt algorithm (Press et al., 1986). $F$ values were calculated by dividing the regression mean square by the residual mean square; the high $F$ values indicate the close fit (all $p<<0.001$ ); the numbers of data points were 392 for curvature of $521 \mathrm{~m}^{-1}$ and 449 for curvature of $172 \mathrm{~m}^{-1}$.

Srinivasan have also addressed the representation of the local shape of an object in the peripheral nerve discharge using sinusoidally shaped steps (LaMotte and Srinivasan 1987a,b; Srinivasan and LaMotte, 1987).

Changing the orientation of the cylinder resulted in a rotation of the SAI population response profile from which the brain could, in principle, easily determine the orientation of the stimulus. From the widths of the profiles in Figure 4 and the lengths of the fingerprints in Figure 8, we estimate that SAIs would be active over an area $>70 \mathrm{~mm}^{2}$, which, at an innervation density of $0.7 \mathrm{~mm}^{-2}$ (Johansson and Vallbo, 1979), comprises about 50 afferents. This is similar to the number of $x-y$ positions used by us in the regressions determining the orientations of the profiles in Table 3; the resolution in our estimates exceeds the 4 or $5^{\circ}$ human difference limens but takes no account of two factors that influence resolution. Noise, particularly in the CNS, is a major factor that increases the uncertainty in any response measure; we do not know the extent of such noise. In addition, not all fibers in the population have the same sensitivity, leading to some "distortion" in the profiles, which could reduce the resolution depending on how the brain accounts for this variation in calculating the orientation. An analogous neural representation of tactile stimulus orientation was demonstrated by LaMotte et al. (1994) for ellipsoids scanned across monkeys' fingerpads; the ellipsoid orientations, presented at $30^{\circ}$ increments, were evident in the spatiotemporal patterns of activity.

There are a number of ancillary tactile cues that could, in principle, assist in the discrimination of orientation. Because the finger is not perfectly flat and isotropic, contact areas with cylinders of different orientation may differ slightly in their lengths, widths, or the pressure distributions within them; these differences would be reflected in differences in the SAI population response length, width, and height. In addition, some members in the population may be active for one orientation but not for another. However, the signal-to-noise ratio for these ancillary cues would be much smaller than for the orientation of the population as a whole, and the resolution would be correspondingly degraded. Moreover, such cues are confounded by the multidimensional nature of the stimulus and could only be used in discrimination tasks in which nothing other than the orientation of the stimulus changed, a situation that is rare in real life. Random changes in the position of the stimulus along the axis of the finger were introduced in our psychophysics experiments in an attempt to eliminate these cues; randomizing the position in the orthogonal direction as well would have provided additional safeguards. For similar reasons we believe that the FAIs and SAIIs do not play a major role in this task. The responses of the FAIs to our stimuli are low in magnitude, but they could contribute to ancillary cues such as the "length" of the response or the location of active units in the population; for the reasons given above, we do not believe that they play a major role in determining stimulus orientation. Similarly, although SAIIs will be activated, including those around the borders of the fingerpads, they are unlikely to provide a robust signal for the orientation of a multidimensional stimulus.

\section{Contact mechanics}

The mechanics of contact between a rigid cylinder and the compliant cylinder-like finger is complex and has an important bearing on neural responses and human performance. Studies of human and monkey skin mechanics have not addressed this issue. The problem is also crucial in robotics, and in this field, there have been some analyses for rigid cylinders contacting elastic 
A. Cylinder $521 \mathrm{~m}^{-1}$

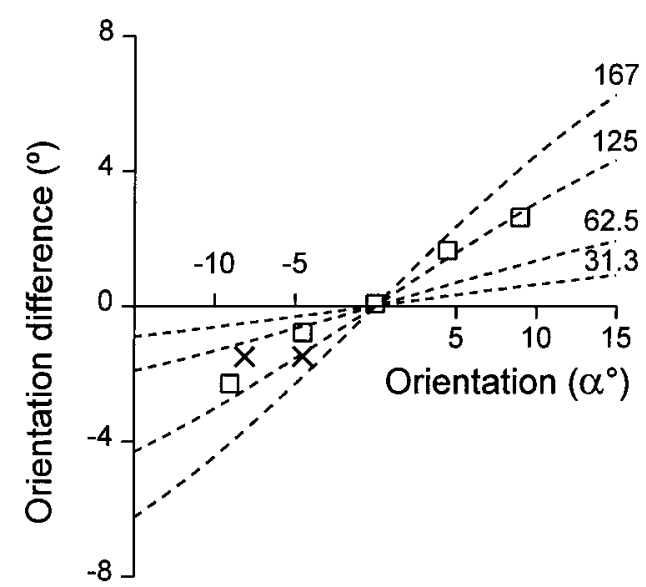

B. Cylinder $172 \mathrm{~m}^{-1}$

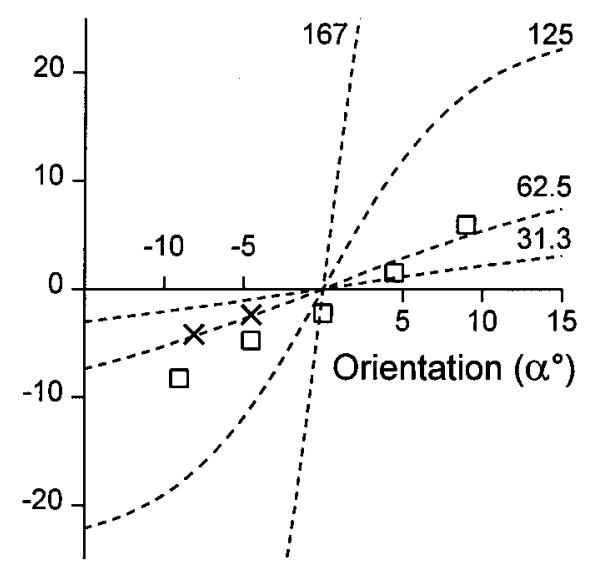

C. Fingerprints

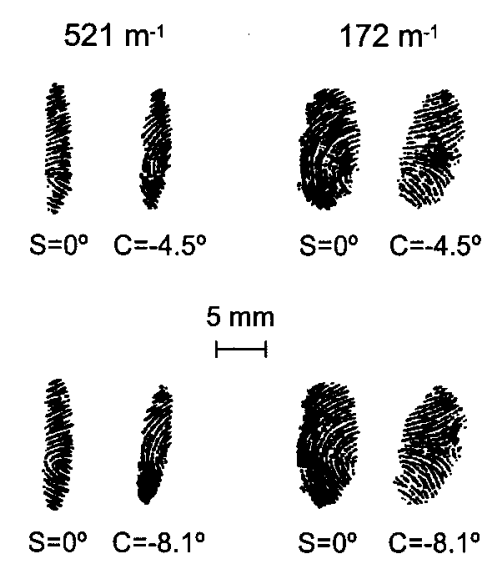

Figure 8. Contact between rigid cylinders and fingers. $A$, B, Broken lines show the difference $(\beta-\alpha)$ between the orientation of the contact ellipse (Fig. $1, \beta)$ and cylinder orientation $(\alpha)$ as a function of cylinder orientation for a rigid cylinder contacting an elastic finger as derived by Fearing and Binford (1991). Calculations are shown for both cylinders used by us (curvatures, 521 and $172 \mathrm{~m}^{-1}$ ) and for fingers ranging in curvature from 31.3 to $167 \mathrm{~m}{ }^{-1}$ (radii ranging from 32 to $6 \mathrm{~mm}$ ). Squares show differences $(\theta-\alpha)$ between SAI response profile orientation $(\theta)$ and cylinder orientation measured in monkeys. Crosses show differences $(\beta-\alpha)$ between contact ellipse orientation and cylinder orientation measured in humans (average of 6 subjects). For clarity, $A$ and $B$ have different ordinate scales. $C$, Fingerprints showing contact areas for a typical human subject. Both cylinders (curvatures, 521 and $172 \mathrm{~m}^{-1}$ ) were used at orientations of $0^{\circ}$ for the standard $(S)$ and -4.5 and $-8.1^{\circ}$ for the comparison $(C)$.

cylinders, which form the fingers of the robots. In particular, the study of Fearing and Binford (1991) showed that the contact area is elliptical, with an orientation that is different from the orientation of the cylinder. The difference in orientation is larger for less curved cylinders and for cylinders that are oriented closer toward the axis of the finger. The situation in the human is even more complex, because the curvature of the finger is not uniform, being flatter at the center of the pad and more curved on the sides of the finger, and also the finger is not a simple elastic body. Nevertheless, currently the robotic analyses provide the best guide to the behavior of human and monkey fingers with regard to the orientation of cylinders. The trends in our data in Figure 8 are consistent with many of the aspects of these simple robotic models. The estimated contact ellipses in the human behaved in a way similar to those in the robot elastic finger, and the same trends were also seen in the orientation of the SAI response profiles recorded in monkeys.

Our data and the model of Fearing and Binford (1991) suggest that human performance (both tactile and manipulative) would be degraded as the orientation of the cylinder deviated further from a line orthogonal to the finger axis, and would be degraded for less curved cylinders. The finding of Lechelt (1992) that the difference limen increased from 10 to $15^{\circ}$ as the cylinder orientation increased from 0 to $45^{\circ}$ is consistent with this hypothesis. However, there are a number of complicating factors. First, the finger is not cylindrical toward the tip but has an appreciable curvature in both directions. This factor probably accounts for the low thresholds $\left(10^{\circ}\right)$ of subjects discriminating orientations close to that of the finger axis in the experiments of Lechelt (1992). Second, during opposition in the human hand, the axes of the thumb and the index finger are not parallel; therefore, cylinders oriented close to the axis of one digit will not be close to the axis of the other digit (Tubiana, 1981). Third, because the SAI population response also contains information about the shape of the cylinder, it is possible that the brain compensates to some degree for the alterations caused by contact mechanics. There is clearly a need for an extension of current primate skin mechanics models (Phillips and Johnson, 1981; Srinivasan, 1989; Srinivasan and Dandekar, 1996) to account for the complex shape of the finger.

\section{Comparison with vision}

Orientation is a crucial parameter of visual stimuli, and there is extensive literature, including human psychophysical studies, single-neuron responses, and robotic vision. The orientation discrimination threshold in humans is different for different stimuli and different experimental conditions. For long lines, the difference limen is of the order of $1^{\circ}$ but varies with the orientation of the stimulus (Orban et al., 1984; Heeley and Buchanan-Smith, 1990; Westheimer and Ley, 1997). Orientation selectivity is a well documented property of cells in the visual cortex, and there are a number of models relating single-cell responses to psychophysical measurements (Paradiso, 1988; Vogels, 1990). In the tactile system, we have only limited data relating the responses of cortical neurons to the orientation of the stimulus (Pubols and Leroy, 1977; Hyvarinen and Poranen, 1978). As yet there are no detailed models for the perception of tactile orientation.

\section{REFERENCES}

Allen PK, Michelman P (1990) Acquisition and interpretation of 3-D sensor data from touch. IEEE Trans Robotics Autom 6:397-404.

Berkemeier M, Fearing R (1993) Determining the axis of a surface of revolution using tactile sensing. IEEE Trans Pattern Anal 15:1079-1087.

Costanzo RM, Gardner EP (1980) A quantitative analysis of responses of direction-sensitive neurons in somatosensory cortex of awake monkeys. J Neurophysiol 43:1319-1341.

Essick GK, Edin BB (1995) Receptor encoding of moving tactile stimuli in humans. II. The mean response of individual low-threshold mechanoreceptors to motion across the receptive field. J Neurosci $15: 848-864$

Essock EA, Krebs WK, Prather JR (1992) An anisotropy of human tactile sensitivity and its relation to the visual oblique effect. Exp Brain Res 91:520-524. 
Fearing RS, Binford TO (1991) Using a cylindrical tactile sensor for determining curvature. IEEE Trans Robotics Autom 7:806-817.

Gandevia SC, McCloskey DI, Burke D (1992) Kinaesthetic signals and muscle contraction. Trends Neurosci 15:62-65.

Goodwin AW (1997) Extracting the shape of an object from the responses of peripheral nerve fibers. In: Neural aspects of tactile sensation (Morley JW, ed). Amsterdam: Elsevier, in press.

Goodwin AW, John KT, Marceglia AH (1991) Tactile discrimination of curvature by humans using only cutaneous information from the fingerpads. Exp Brain Res 86:663-672.

Goodwin AW, Browning AS, Wheat HE (1995) Representation of curved surfaces in responses of mechanoreceptive afferent fibers innervating the monkey's fingerpad. J Neurosci 15:798-810.

Hahn H (1994) Recognition of 3D objects by a 3-fingered robot hand equipped with tactile and force sensors. IEEE Int Conf MFI 297-304.

Heeley DW, Buchanan-Smith HM (1990) Recognition of stimulus orientation. Vision Res 30:1429-1437.

Hyvarinen J, Poranen A (1978) Movement-sensitive and direction and orientation-selective cutaneous receptive fields in the hand area of the post-central gyrus in monkeys. J Physiol (Lond) 283:523-537.

Johansson RS (1996) Sensory and memory information in the control of dextrous manipulation. In: Neural bases of motor behaviour (Lacquaniti F, Viviani P, eds), pp 205-260. Dordrecht, Netherlands: Kluwer.

Johansson RS, Vallbo AB (1979) Tactile sensibility in the human hand: relative and absolute densities of four types of mechanoreceptive units in glabrous skin. J Physiol (Lond) 286:283-300.

Johnson KO (1980) Sensory discrimination: decision process. J Neurophysiol 43:1771-1792.

Keyson DV, Houtsma AJ (1995) Directional sensitivity to a tactile point stimulus moving across the fingerpad. Percept Psychophys 57:738-744.

Kops CE, Gardner EP (1996) Discrimination of simulated texture patterns on the human hand. J Neurophysiol 76:1145-1165.

LaMotte RH, Srinivasan MA (1987a) Tactile discrimination of shape: responses of slowly adapting mechanoreceptive afferents to a step stroked across the monkey fingerpad. J Neurosci 7:1655-1671.

LaMotte RH, Srinivasan MA (1987b) Tactile discrimination of shape: responses of rapidly adapting mechanoreceptive afferents to a step stroked across the monkey fingerpad. J Neurosci 7:1672-1681.

LaMotte RH, Srinivasan MA (1993) Responses of cutaneous mechanoreceptors to the shape of objects applied to the primate fingerpad. Acta Psychol (Amst) 84:41-51.

LaMotte RH, Srinivasan MA (1996) Neural encoding of shape: responses of cutaneous mechanoreceptors to a wavy surface stroked across the monkey fingerpad. J Neurophysiol 76:3787-3797.

LaMotte RH, Srinivasan MA, Lu C, Klusch-Petersen A (1994) Cutaneous neural codes for shape. Can J Physiol Pharmacol 72:498-505.

Lechelt EC (1992) Tactile spatial anisotropy with static stimulation. Bull Psychonomic Soc 30:140-142.

Macmillan NA, Creelman CD (1991) Detection theory: a user's guide. Cambridge, UK: Cambridge UP.
Orban G, Vandenbussche E, Vogels R (1984) Human orientation discrimination tested with long stimuli. Vision Res 24:121-128.

Paradiso MA (1988) A theory for the use of visual orientation information which exploits the columnar structure of striate cortex. Biol Cybern 58:35-49.

Phillips JR, Johnson KO (1981) Tactile spatial resolution. III. A continuum mechanics model of skin predicting mechanoreceptor responses to bars, edges, and gratings. J Neurophysiol 46:1204-1225.

Press WH, Flannery BP, Teukolsky SA, Vetterling WT (1986) Numerical recipes. The art of scientific computing. Cambridge, UK: Cambridge UP.

Pubols LM, Leroy RF (1977) Orientation detectors in the primary somatosensory neocortex of the raccoon. Brain Res 129:61-74.

Reynaerts D, van Brussel H (1993) Tactile sensing data interpretation for object manipulation. Sensor Actuat A37-38:268-273.

Ruiz S, Crespo P, Romo R (1995) Representation of moving tactile stimuli in the somatic sensory cortex of awake monkeys. J Neurophysiol 73:525-537.

Schneider SL, Hughes B, Epstein W, Bach-y-Rita P (1986) The detection of length and orientation changes in dynamic vibrotactile patterns. Percept Psychophys 40:290-300.

Srinivasan MA (1989) Surface deflection of primate fingertip under line load. J Biomech 22:343-349.

Srinivasan M, Dandekar K (1996) Investigation of the mechanics of tactile sense using two-dimensional models of the primate fingertip. J Biomech Eng Trans ASME 118:48-55.

Srinivasan MA, LaMotte RH (1987) Tactile discrimination of shape: responses of slowly and rapidly adapting mechanoreceptive afferents to a step indented into the monkey fingerpad. J Neurosci 7:1682-1697.

Stevens JC, Patterson MQ (1995) Dimensions of spatial acuity in the touch sense: changes over the life span. Somatosens Mot Res 12:29-47.

Tubiana R (1981) The hand. Philadelphia: Saunders.

Vallbo AB, Johansson RS (1984) Properties of cutaneous mechanoreceptors in the human hand related to touch sensation. Hum Neurobiol 3:3-14.

Van Boven RW, Johnson KO (1994) A psychophysical study of the mechanisms of sensory recovery following nerve injury in humans. Brain 117:149-167.

Vogels R (1990) Population coding of stimulus orientation by striate cortical cells. Biol Cybern 64:25-31.

Warren S, Hamalainen HA, Gardner EP (1986) Objective classification of motion- and direction-sensitive neurons in primary somatosensory cortex of awake monkeys. J Neurophysiol 56:598-622.

Westheimer G, Ley EJ (1997) Spatial and temporal integration of signals in foveal line orientation. J Neurophysiol 77:2677-2684.

Wheat HE, Goodwin AW, Browning AS (1995) Tactile resolution: peripheral neural mechanisms underlying the human capacity to determine positions of objects contacting the fingerpad. J Neurosci 15:5582-5595. 